\title{
Editorial
}

\section{Influência e controle da aquiescência na análise fatorial}

DOI: 10.15689/ap.2017.1602.ed

Estudos de evidências de validade de estrutura interna são cruciais para o desenvolvimento de instrumentos psicométricos. Além de proporcionarem aferição de qualidades técnicas a esses instrumentos, potencializam a consolidação de redes nomológicas entre os construtos psicológicos. Contudo, para instrumentos de autorrelato, as estimativas dos parâmetros do modelo podem sofrer influência de vieses associados às respostas dos sujeitos (Danner, Aichholzer, \& Rammstedt, 2015; Maydeu-Olivares \& Coffman, 2006; Soto, John, Gosling, \& Potter, 2008). Nesse sentido, ao responder a um item por meio de uma escala do tipo Likert, por exemplo, uma parte da resposta do sujeito pode ser, hipoteticamente, atribuída à maneira como aquele sujeito específico responde aos itens, além do fator latente. Entre as diversas fontes de viés de resposta, destaco, neste editorial, a aquiescência.

A aquiescência diz respeito à tendência de responder positivamente aos itens, a despeito do seu conteúdo descritivo (Billiet \& McClendon, 2000). Para itens do tipo Likert, com categorias de resposta variando de "discordo fortemente" a "concordo fortemente", padrões de resposta aquiescentes podem ser observados pela alta frequência de endosso da categoria "concordo fortemente", ainda que os conteúdos descritivos dos itens sejam, por vezes, opostos. Por exemplo, os itens "sou otimista" e "sou pessimista" englobam o mesmo conteúdo descritivo de personalidade, mas em polos opostos. Assim, nesse exemplo, esperar-se-ia o endosso de categorias opostas, caso as respostas a esse par de itens fossem influenciadas unicamente pelo conteúdo descritivo (por exemplo, categoria "concordo fortemente" para o item "sou otimista" e categoria "discordo fortemente" para o item "sou pessimista").

Contudo, escalas Likert também são influenciadas pela tendência de concordar (Billiet \& McClendon, 2000), o que é definido por aquiescência ou "dizer sempre sim" ("yea-saying"). Ou, no fenômeno oposto, pela desaquiescência. No exemplo sobre o otimismo, a aquiescência reflete-se em uma tendência de endossar a categoria "concordo fortemente" do item "sou otimista" e, na mesma coleta, endossar as categorias "neutro" ou "concordo" do item "sou pessimista".

O viés de resposta, se não controlado, pode resultar na estimação de um fator comum geral espúrio e não associado ao conteúdo descritivo dos itens, além de comprometer a interpretabilidade da estrutura interna do instrumento (Billiet \& McClendon, 2000; Danner et al., 2015; Maydeu-Olivares \& Coffman, 2006; Rammstedt \& Farmer, 2013). Ademais, a aquiescência tende a enviesar as correlações com outras variáveis (Mirowsky \& Ross, 1991).

Entretanto, existem métodos bem interessantes para o controle desse tipo de viés. Entre os quais, ressalto dois: ipsatização (Soto et al., 2008; Ten Berge, 1999) e modelagem de interceptos randômicos (Maydeu-Olivares \& Coffman, 2006). Esses métodos pressupõem que a escala seja composta de itens em ambos os polos do construto (isto é, itens positivos e negativos, como, por exemplo, "otimista" e "pessimista").

A ipsatização, ou padronização intrassujeitos, é um método bastante simples e envolve a recodificação dos escores brutos com base na média e no desvio padrão (DP) dos itens positivos e negativos de cada sujeito. Para tanto, utiliza-se a equação: [(escore bruto - média sujeito) / DP sujeito]. Ressalta-se que a média e o DP se referem aos escores brutos dos itens positivos e negativos de cada sujeito, e não de uma variável específica. Também é possível utilizar uma variação do procedimento na qual os escores brutos são recodificados apenas levando em conta a média, desconsiderando o DP (uma espécie de centralização intrassujeitos). Após a recodificação, as médias dos itens recodificados serão iguais entre todos os participantes (caso se tenha ponderado o DP na recodificação, este também será igual entre todos os participantes). Assim, o escore total não pode ser mais comparado entre os sujeitos, e, por esse motivo, esse tipo de recodificação é denominado ipsatização. O procedimento tem apresentado bons resultados, melhorando a interpretabilidade da estrutura interna dos instrumentos (Soto et al., 2008; Ten Berge, 1999).

O segundo procedimento, de interceptos randômicos, envolve a modelagem de um fator geral não correlacionado aos fatores de conteúdo descritivo, conforme Figura 1 (Maydeu-Olivares \& Coffman, 2006). Os oito itens 
(representados por quadrados, sendo quatro positivos e quatro negativos) são explicados por dois fatores de conteúdo descritivo (F1 e F2). Como todas as cargas do "fator intercepto" são fixadas em 1, o escore estimado desse fator pode ser interpretado como uma espécie de "escore individual de dificuldade", ou "escore de aquiescência". Ou seja, o fator reflete em que medida os indivíduos estão propensos a concordar com o item, independentemente do conteúdo. A vantagem desse método é que os escores dos fatores de conteúdo descritivo não são ipsatizados (mesmo para modelos com fator geral de conteúdo). Uma variação desse modelo é possível relaxando a correlação fixa em 0 entre o intercepto e os fatores de conteúdo descritivo. Essa variação implica impactos distintos do viés de resposta nos diferentes fatores, o que precisa ser muito bem explicado teoricamente.

No presente editorial, buscou-se ilustrar a utilização dos métodos de ipsatização e de interceptos randômicos, comparando a performance de análises fatoriais (análise fatorial exploratória - AFE, e análise fatorial confirmatória AFC) em modelagens com e sem o controle da aquiescência. Para tanto, foi simulado um banco de dados cuja estrutura fatorial era conhecida a priori. Foram simuladas as respostas de 1.000 participantes para 12 itens ordinais (escala Likert de cinco pontos). A estrutura do modelo foi composta de três fatores de conteúdo descritivo e um fator de intercepto randômico. As cargas fatoriais foram especificadas entre 0,30 e 0,40 , e as correlações entre os fatores, em 0,40 , mantendo o modelo próximo de um cenário comum na Psicologia: cargas fatoriais baixas e correlações moderadas entre os fatores. As cargas do fator de intercepto randômico foram fixadas em 0,20 , para que na modelagem esse fator contribuísse menos na explicação da variância dos itens se comparado aos fatores de conteúdo descritivo. As análises e simulação foram realizadas por meio do Lavaan do R, programas Factor 10.4 e Mplus 7.11. Em todas as análises fatoriais, os itens foram declarados como categóricos ordinais, e utilizaram-se matrizes de correlações policóricas. Os itens recodificados por ipsatização foram arredondados para manter a natureza ordinal dos dados. Os parâmetros dos itens foram estimados por weighted least squares means and variance adjusted (WLSMV) e unweighted least squares (ULS).

Para os escores brutos, os resultados dos métodos de retenção fatorial sugeriram a extração entre um e quatro fatores. A análise paralela (AP) (Horn, 1965) com permutação indicou um fator; a análise paralela clássica apontou quatro fatores; Minimum Average Partial (MAP) (Velicer, 1976) indicou uma dimensão; e Hull (Lorenzo-Seva, Timmerman, \& Kiers, 2011) sugeriu a extração de quatro dimensões. Recentemente, Golino e Epskamp (2016) propuseram um método de decisão com base em análise de redes denominado de exploratory graphic analysis (EGA). Esse método sugeriu a extração de duas dimensões: uma para os itens positivos e outra para os itens negativos.

Para os escores recodificados por ipsatização, Hull e análise paralela indicaram a extração de três dimensões. O EGA apontou a extração de quatro dimensões, contudo uma inspeção visual do gráfico de rede (não disponibilizado aqui por falta de espaço) mostrou, claramente, três agrupamentos de itens. Além disso, após a inversão dos itens negativos (e ipsatizados), o EGA apontou para a extração de três fatores, conforme o modelo simulado. MAP sugeriu extrair uma dimensão apenas.

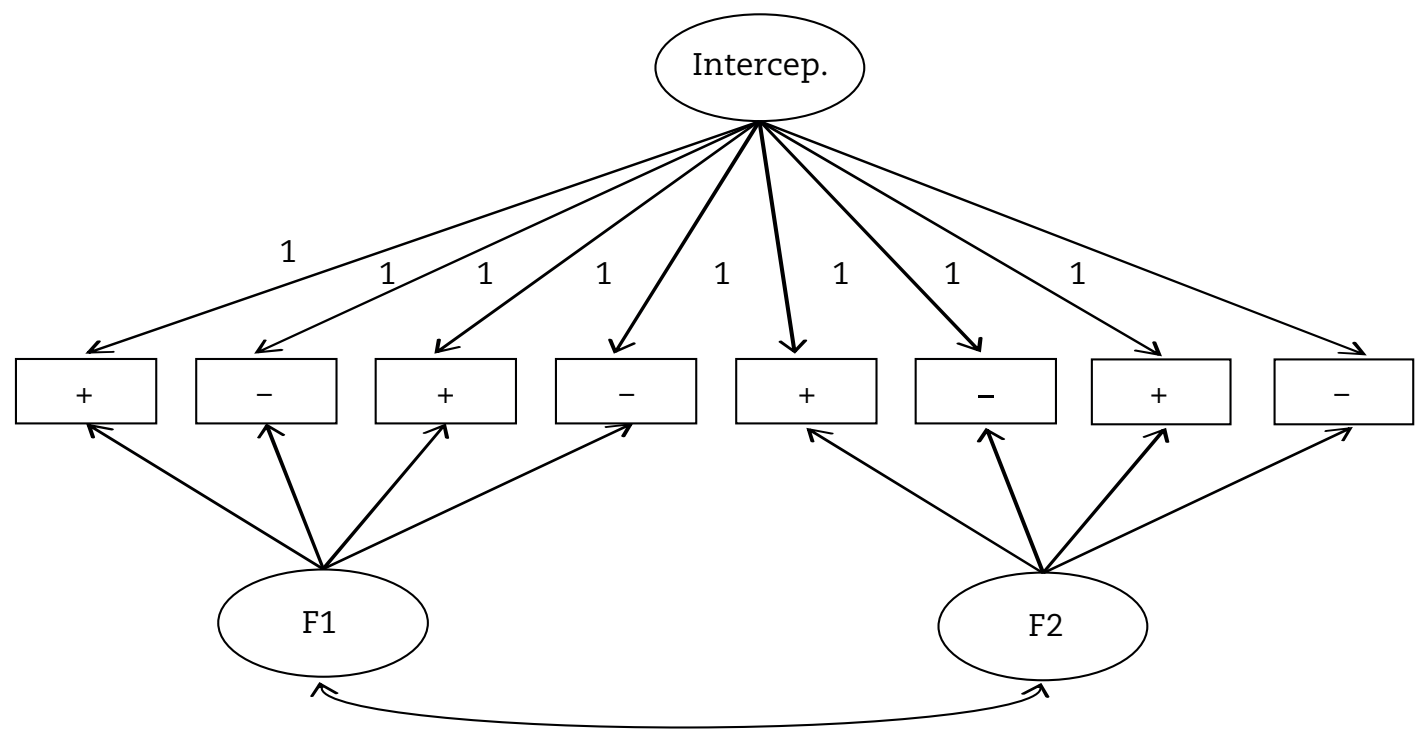

Nota: F1=fator 1; F2=fator 2; Intercep.=fator intercepto randômico; $+=$ item no polo positivo do fator latente; -=item no polo negativo do fator latente.

Figura 1. Modelo de interceptos randômicos 
Os resultados indicaram baixa performance dos métodos de extração com os dados brutos, sem o controle do viés de resposta. Para os escores ipsatizados, ainda que os métodos de extração tenham apresentado falhas, AP, Hull e EGA indicaram adequadamente o número de dimensões. Em suma, a decisão do número de fatores a serem extraídos fica seriamente comprometida nos dados enviesados pela aquiescência, caso esse viés não seja controlado. Para ampliar a discussão, submeti os dados brutos e os recodificados a análises fatoriais exploratórias e confirmatórias cujas cargas fatoriais são exibidas da Tabela 1.

As análises fatoriais com os dados brutos, mesmo utilizando correlações policóricas, falharam completamente na recuperação do modelo verdadeiro (Tabela 1). Tais análises sequer indicaram adequadamente o polo do conteúdo da maior parte dos itens. No entanto, quando controlado o viés comum dos itens, tanto a AFE com os escores recodificados por ipsatização quanto a $\mathrm{AFC}$ com interceptos randômicos reproduziram adequadamente o modelo verdadeiro. Por fim, analisei os modelos de estrutura, expostos na Tabela 1, por meio de equações estruturais, e os indicadores de ajuste estão na Tabela 2.

Conforme esperado, os modelos indicados na AFE para os dados brutos não se ajustaram bem aos dados quando analisados por AFC. Por sua vez, os modelos com controle de viés de resposta (fator comum) ajustaram-se muito bem aos dados (incluindo qui-quadrado não significativo). Isso era esperado para o modelo de interceptos randômicos, uma vez que os dados foram simulados tendo esse modelo como verdadeiro. Não obstante, a performance da ipsatização é bastante surpreendente. Tal resultado também pode indicar que os dados foram simulados de maneira a apresentar, de fato, um fator comum de viés de resposta. Removido esse fator comum dos dados, mesmo por ipsatização, a estrutura verdadeira é revelada.

Tabela 1

Análises fatoriais dos dados brutos (com e sem o controle de interceptos randômicos) e dos recodificados por ipsatização

\begin{tabular}{|c|c|c|c|c|c|c|c|c|c|c|c|c|c|c|c|c|}
\hline & \multirow{2}{*}{\multicolumn{2}{|c|}{$\begin{array}{l}\text { Dados } \\
\text { modelados }\end{array}$}} & \multicolumn{10}{|c|}{ AFE } & \multirow{2}{*}{\multicolumn{4}{|c|}{$\begin{array}{c}\text { AFC } \\
\text { Interceptos } \\
\text { randômicos } \\
\end{array}$}} \\
\hline & & & \multicolumn{3}{|c|}{$\begin{array}{l}\text { Dados } \\
\text { brutos }\end{array}$} & \multicolumn{4}{|c|}{$\begin{array}{l}\text { Dados } \\
\text { brutos }\end{array}$} & \multicolumn{3}{|c|}{$\begin{array}{l}\text { Escore ipsatizado } \\
\text { pela média }\end{array}$} & & & & \\
\hline & $\begin{array}{c}\text { Polo } \\
\text { descritivo }\end{array}$ & Fator & F1 & F2 & F3 & F1 & F2 & F3 & F4 & F1 & F2 & F3 & F1 & F2 & F3 & $\begin{array}{c}\mathrm{F} \\
\text { Inter }\end{array}$ \\
\hline Item 1 & + & F1 & & $-0,32$ & & 0,55 & & & & 0,51 & & & 0,52 & & & 0,23 \\
\hline Item 2 & + & F1 & & & 0,32 & 0,50 & & & & 0,48 & & & 0,51 & & & 0,23 \\
\hline Item 3 & - & F1 & & 0,56 & & $-0,44$ & & & & $-0,60$ & & & $-0,52$ & & & 0,23 \\
\hline Item 4 & - & F1 & & 0,50 & & $-0,34$ & & & & $-0,39$ & & & $-0,47$ & & & 0,23 \\
\hline Item 5 & - & F2 & 0,39 & & & & & & 0,44 & & $-0,42$ & & & $-0,38$ & & 0,23 \\
\hline Item 6 & + & F2 & & & 0,51 & & & 0,45 & & & 0,44 & & & 0,44 & & 0,23 \\
\hline Item 7 & - & F2 & 0,38 & & & & & & 0,45 & & $-0,44$ & & & $-0,39$ & & 0,23 \\
\hline Item 8 & + & F2 & & & 0,47 & & & 0,41 & & & 0,41 & & & 0,45 & & 0,23 \\
\hline Item 9 & + & F3 & & & & & & & & & & 0,34 & & & 0,30 & 0,23 \\
\hline Item 10 & - & F3 & & & & & 0,44 & & & & & $-0,49$ & & & $-0,33$ & 0,23 \\
\hline Item 11 & - & F3 & & & & & 0,36 & & & & & $-0,34$ & & & $-0,35$ & 0,23 \\
\hline Item 12 & + & F3 & & & & & & & & & & 0,32 & & & 0,40 & 0,23 \\
\hline
\end{tabular}

Nota: Todos os itens foram modelados para terem carga de 0,20 no fator representativo do viés de aquiescência. AFE=análise fatorial exploratória; $\mathrm{AFC}=$ análise fatorial confirmatória; F Inter=fator intercepto randômico.

Tabela 2

Indicadores de ajuste das análises fatoriais confirmatórias

\begin{tabular}{lcccc}
\hline & $\chi 2(\mathrm{gl})$ & CFI & TLI & RMSEA (IC90\%) \\
\hline 3 fatores (dados brutos) & $126,89(31)^{*}$ & 0,85 & 0,79 & $0,06(0,05-0,07)$ \\
4 fatores (dados brutos) & $189,64(47)^{*}$ & 0,81 & 0,73 & $0,06(0,05-0,07)$ \\
3 fatores (escores ipsatizados) & $47,56(51)$ n.s. & 1,00 & 1,01 & $0,00(0,00-0,02)$ \\
Intercepto Randômico & 40,79 (50) n.s. & 1,00 & 1,02 & $0,00(0,00-0,01)$ \\
\hline
\end{tabular}

Nota: * $p<0,05 ;$ n.s=estatisticamente não significativo; CFI=comparative fit index; TLI=Tucker Lewis index; RMSEA=root mean square error of approximation. 
O presente editorial buscou ilustrar o potencial do estrago do viés de resposta e a importância do uso de delineamentos de pesquisa que possam minimizar os efeitos desse problema. Sem o devido controle, o viés de resposta pode levar o pesquisador a adotar soluções fatoriais espúrias. Não obstante, na prática, os modelos não são tão perfeitos como os simulados, e dificilmente um modelo quase perfeito será revelado na primeira coleta de dados. No entanto, quanto mais refinado e planejado for o delineamento do estudo, maiores são as chances de os modelos reproduzirem a estrutura interna adequada.

Ressalto que este editorial não tem a pretensão de relatar um estudo de simulação stricto sensu, o que exigiria mais controle e variabilidade das situações, tais como diferentes valores de cargas fatoriais e correlações entre os fatores. Além disso, a simulação do viés de resposta foi operacionalizada por meio de um modelo com uma dimensão geral cujas relações com os itens são todas positivas e de mesma magnitude (carga fatorial) tanto para os itens positivos quanto para os negativos. Essa ideia técnica é perfeitamente compatível com o conceito de viés de resposta por aquiescência. Contudo, é possível que outros tipos de vieses também tenham sido englobados nessa dimensão geral.

Por fim, o editorial também tem a finalidade de incentivar os pesquisadores a submeterem manuscritos que relatem estudos com delineamentos para controle de vieses, especialmente aqueles que buscam evidenciar a estrutura interna dos escores de instrumentos. Tais manuscritos poderão melhorar substancialmente a qualidade dos instrumentos e das pesquisas da nossa área.

Felipe Valentini

Editor Associado

Universidade Salgado de Oliveira, Niterói-RJ, Brasil

\section{Referências}

Billiet, J. B., \& McClendon, M. J. (2000). Modeling acquiescence in measurement models for two balanced sets of items. Structural Equation Modeling, 74, 608-628. doi: 10.1207/S15328007SEM0704

Danner, D., Aichholzer, J., \& Rammstedt, B. (2015). Acquiescence in personality questionnaires: Relevance, domain specificity, and stability. Journal of Research in Personality, 57, 119-130. doi: 10.1016/j.jrp.2015.05.004

Golino, H., \& Epskamp, S. (2016). Exploratory graph analysis: A new approach for estimating the number of dimensions in psychological research. arXiv:1605.02231. Retrieved from https://arxiv.org/abs/1605.02231

Horn, J. (1965). A rationale and test for the number of factors in factor analysis. Psychometrika, 30, 179-185. doi: 10.1007/BF02289447

Lorenzo-Seva, U., Timmerman, M. E., \& Kiers, H. A. L. (2011). The Hull Method for Selecting the Number of Common Factors. Multivariate Behavioral Research, 46, 340-364. doi: 10.1080/00273171.2011.564527

Maydeu-Olivares, A., \& Coffman, D. L. (2006). Random intercept item factor analysis. Psychological Methods, 11, 344-362. doi: 10.1037/1082989X.11.4.344

Mirowsky, J., \& Ross, C. E. (1991). Elminating defence and agreement bias from measures of the sense of control: A 2 x 2 Index. Social Psychology Quarterly, 54, 127-145. doi: 10.2307/2786931

Rammstedt, B., \& Farmer, R. F. (2013). The impact of acquiescence on the evaluation of personality structure. Psychological Assessment, 25, 1137-1147.

Soto, C. J., John, O. P., Gosling, S. D., \& Potter, J. (2008). The developmental psychometrics of big five self-reports: Acquiescence, factor structure, coherence, and differentiation from ages 10 to 20. Journal of Personality and Social Psychology, 94, 718-737. doi: 10.1037/00223514.94.4.718

Ten Berge, J. M. F. (1999). A legitimate case of component analysis of ipsative measures, and partialling the mean as an alternative to ipsatization. Multivariate Behavioral Research, 34, 89-102.

Velicer, W. (1976). Determining the number of components from the matrix of partial correlations. Psychometrika, 41, 321-327. doi: 10.1007/ BF02293557 